\title{
Unusual Spreading of Colon Adenocarcinoma into the Right Hip Area: A Case Report
}

\author{
Renata Dobrila Dintinjana ${ }^{1 *}$, Duška Petranović ${ }^{2}$, Gorazd Pilčič ${ }^{2}$, Vjekoslav Ilijic ${ }^{3}$, Davor Petranović ${ }^{4}$, \\ Marijan Dintinjana ${ }^{5}$ \\ ${ }^{1}$ Department of Oncology, University Hospital Center Rijeka, Rijeka, Croatia; ${ }^{2}$ Department of Internal Medicine, University Hospital \\ Center Rijeka, Rijeka, Croatia; ${ }^{3}$ Department of Internal Medicine, Salem Hospital, Bern, Switzerland; ${ }^{4}$ Department of Radiology, \\ Medico Polyclinic, Rijeka, Croatia; ${ }^{5}$ Private Clinic of Dr. Dintinjana, Rijeka, Croatia. \\ Email: *renatadobrila@windowslive.com
}

Received September $29^{\text {th }}, 2012$; revised October $28^{\text {th }}, 2012$; accepted November $8^{\text {th }}, 2012$

\begin{abstract}
Adenocarcinoma is the most common colon cancer type. This form of colonic neoplasms usually metastasizes initially to regional lymphatic system and through the blood circulation to the lungs and liver, while other forms of expansion and involvement of other organ systems are less common. Extraluminal carcinoma is very rare. Only a few authors describe the direct spread of cancer to adjacent structures and organs. In this paper we present a case of a 76-year old patient with tumor mass extending from the colon to the right hip area "per continuitatem". The patient had no symptoms and signs that would indicate the presence of neoplastic process in the colon. Only a discomfort in right leg was present and finally tumor mass was visualized. Biopsy and patohistology findings confirmed final diagnosis and type of tumor in the right hip region. Pathohistologicaly tumor was adenocarcinoma.
\end{abstract}

Keywords: Colon Adenocarcinoma; Local Spreading; Right Hip

\section{Introduction}

In Western countries, cancer of the large intestine and rectum is the second most common type of cancer and the second leading cause of cancer death. Colorectal cancer it is the most common cause of cancer death among non-smokers. Factors associated with increased risk of colorectal cancer are host susceptibility and a sequence of different carcinogenic exposures. Although specific etiology for sporadic colorectal cancer is still elusive predisposing hereditary and environmental factors have been clearly identified [1].

Almost all colorectal cancers are adenocarcinomas, which develop from the lining of the colon and rectum. It usually begins on the surface of the intestinal or rectal lining or on a polyp. As the cancer grows, it begins to invade the wall of the intestine or rectum, and continuously spread to the nearby lymph nodes and perirectal fat tissue. Also, it metastasizes by blood and lymph vessels mainly to the liver, or other sites [2]. Extraluminal carcinomas are very rare and they can produce unexpected manifestations of disease. Very few authors described local invasion with colorectal cancer to the nearby structures like invasion of inguinal structures $[3,4]$.

*Corresponding author.

\section{Case Report}

A 76-year old patient was admitted to the Department of Internal medicine, University Clinical Hospital Center Rijeka, due to progressive fatigue for the last two months.

At admission patient was afebrile, eupnoic, cardiorespiratory functions sufficient. There were no palpabile lymph nodes. Liver and spleen were not palpable. Her appetite was normal, had no weight loss, nausea and vomiting and did not noticed any changes in stool colour, frequency or consistency. She had no any abdominal discomfort or pain. Also urine and stool was normal.

Physical status was normal but she complained on slight discomfort in the right leg. The leg was practically normal, but when measured in hip area, circumference was $5 \mathrm{~cm}$ larger than the left leg.

Laboratory analysis were completely normal (Hemoglobin level was $12.5 \mathrm{~g} / \mathrm{dL}$, leukocytes 4.5 , thrombocytes $238 \times 10^{-9} / \mathrm{L}$, with normal biochemical parameters including liver enzimes, protein electrophoresis, beta $2 \mathrm{mi}-$ croglobulin). There were no occult bleeding in the stool, and urine analyses were completely normal. We suspected paraneoplastic syndrome and performed mamography, gynecological exam, cardiology and pulmology consultation which were all normal.

As patient at admission complained on discomfort in 
the right leg we performed ultrasonography of the soft tissue and find soft tissue tumour infiltrate in the upper hip with no clear borders, in the region of quadriceps femoris more than $20 \mathrm{~cm}$ long.

Figure 1. Ultrasonography of the right hip. Infiltration with no clear borders in femoral region infiltrates musculus quadriceps femoris.

We analyzed tumour markers (Ca 125, Ca 19-9, AFP, CEA, Ca 15-3, Cyfra) and carcinoembryonic antigen (CEA) was very high (more than 5 times above normal values). We performed total colonoscopy but no tumour has been visualized.

To confirm the existence of the tumor mass computerized tomography (CT) scan of the pelvis and right thigh region was performed. CT findings showed a tumor mass spreading through soft tissue, lower pelvis and upper femoral region to the level of lower third of femur (about $26 \mathrm{~cm}$ in longer diameter) and infiltrate $2 / 3$ of leg circumference.

Figure 2. CT scan of the pelvis and right femoral region. CT scan pictures show tumour mass spreading through soft tissue from abdomen, lower pelvis through upper femoral region to the level of lower third of femur (about $26 \mathrm{~cm}$ in longer diameter) and infiltrate $2 / 3$ of leg circumference.

Biopsy of the femoral region soft tissue has been made and pathohistological analysis revealed unusual finding. Microscopically, the specimen consisted of tumor tissue in the form of atypical glandular and cribriform formations, as well as solid clusters and single tumor cells set in fibrous stroma. Tumor glands were lined with atypical pseudostratified cylindrical epithelium, reminiscent of colonic epithelium. The results of routine microscopical and immunohistochemical staining (CK20+, CK7-, CEA+) were the most consistent with the diagnosis of colonic adenocarcinoma.

Figure 3. Microscopic and immunohistochemical finding of the adenocarcinoma. Microscopical picture of metastatic adenocarcinoma stained routinely (A) and immunohistochemically with antibodies against citokeratin 20 (B), citokeratin 7 (C) and carcinoembrional antigen (D).

Unfortunately the patient deteriorated quickly with swelling and painful leg, formation of solid, hard tumor mass, and signs of phlebothrombosis. Although the best supportive care was applied, she died three weeks after admission to hospital.

\section{Discussion}

We present the very unusual case of extraluminal colon carcinoma spreading from the abdomen through the right hip area "per continuitatem". To the best of our knowledge, we didn't find similar case published in the literature, where extraluminal colonic adenocarcinoma directly invaded into the hip [5-13].

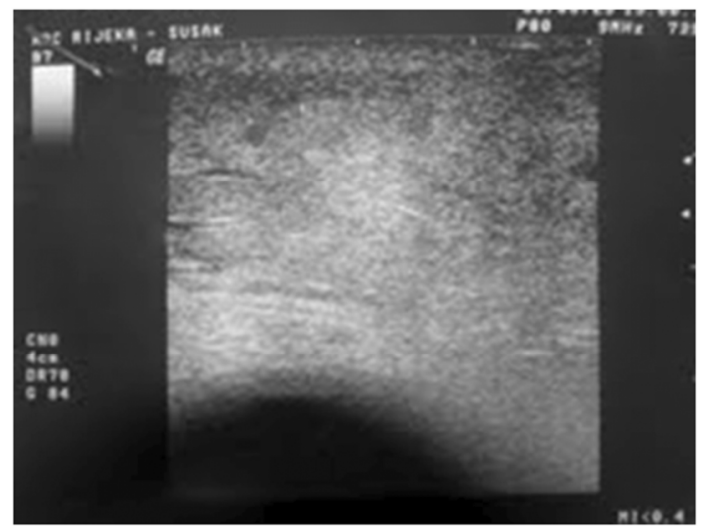

Figure 1. Ultrasonography of the right hip. Infiltration with no clear borders in femoral region infiltrates musculus quadriceps femoris.

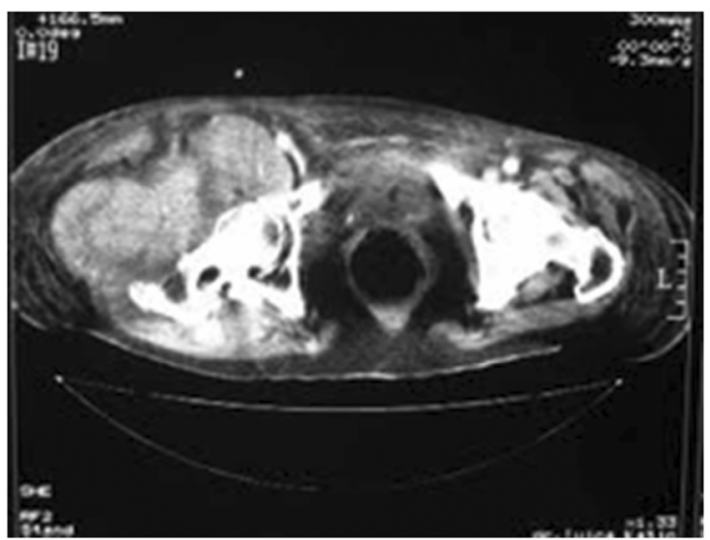

Figure 2. CT scan of the pelvis and right femoral region. CT scan pictures show tumour mass spreading through soft tissue from abdomen, lower pelvis through upper femoral region to the level of lower third of femur (about $26 \mathrm{~cm}$ in longer diameter) and infiltrate 2/3 of leg circumference.
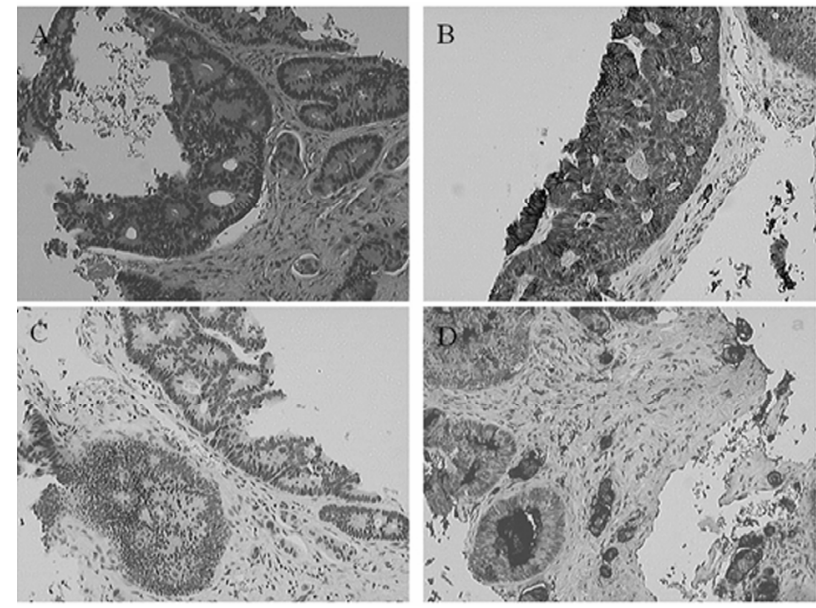

Figure 3. Microscopic and immunohistochemical finding of the adenocarcinoma. Microscopical picture of metastatic adenocarcinoma stained routinely (A) and immunohistochemically with antibodies against citokeratin 20 (B); citokeratin 7 (C) and carcinoembrional antigen (D). 
Our patient did not have any sign of distant metastasis in the same time when local tumor was more than $20 \mathrm{~cm}$. The tumor presented in unusual way and patient also had no any sign of usually colon cancer symptoms related to its local presence in the large bowel or by its effect on other organs if it has spread (a change in bowel habit blood in the stool, bloating, persistent abdominal distention, constipation, feeling of fullness abdominal discomfort, unexplained weight loss, nausea and vomiting). The only but unspecific symptom was persistent, chronic fatigue. Extraluminal colorectal cancers have unusual presentation. Discomfort and swelling and later painful leg led us to diagnosis.

Explanation of this pattern of spreading is very difficult. When cells lining the colon become malignant, they first grow locally and may invade partially or totally through the wall of the bowel and into adjacent structures and organs. But usually in this process, when the tumor penetrate and invade the lymphatics or the capillaries locally and it gains access to the circulation, malignant cells work their way to other areas of the body and metastases in the regional lymph nodes or distant from the primary tumor, in the liver, bones and other sites.

Mechanisms of local spreading are not fully understood. But previous researches have pointed to 3 ways that the tumour is most likely to do this:

Ad. 1. Pressure from the growing tumour force its way through some paths easier than others, for instance between sheets of muscle tissue rather than straight through bone or cartilage. A cancer probably just grows out in a random direction from the place where it started spreading into some tissues more easily than others. For example, large blood vessels that have very strong walls and dense tissues such as cartilage are hard for tumours to grow into. So locally, tumours probably just take the easiest route.

As the cancer grows, it will squeeze and block small blood vessels in the area. Deprived of blood and oxygen, some of the normal tissue will begin to die. This will make it easier for the tumour to continue to push its way through.

Ad. 2. Growing tumours seem to have high levels of enzymes that break down tissue. These enzymes are likely to make it easier for the tumour to forge a path through the healthy tissue.

Ad. 3. Actively moving through the tissue one of the properties of cancer cells that makes them different from normal cells is that they can move about more easily. So it seems likely that one of the ways that cancers spread through nearby tissues is by direct tumor cells movement.

A particular tumor will probably use all three of these ways of spreading. Which way is used most will depend partly on the type of tumor, and partly on where in the body it is growing [4]. Treatment options have been disccussed. Surgery approach was impossible due to large mass with unclear borders position of tumor and general patient's condition. Regular chemotherapy for locally advanced colorectal cancer (Folfiri, Xeliri, Folfox, Xelox + bevacizumab) seems too aggressive for patient's condition, and objective response rate for locally advanced colorectal carcinoma is low [14]. Radiotherapy was option for palliative purposes to shrinkage the mass of tumor and to diminish discomfort patient felt in invaded leg. Our atient deteriorated quickly and radiotherapy was not performed.

We conclude that extraluminal colorectal cancer is rare condition producing unusual clinical manifestations Physician must establish diagnosis, searching unusual tumors on unusual places, using all modern diagnostic techniques.

\section{REFERENCES}

[1] R. Dobrila-Dintinjana, D. Trivanović, M. Dintinjana, J. Vukelić and N. Vanis, "Effects of Dietary Counseling on Patients with Colorectal Cancer," In: R. Etarh, Ed., Colorectal Cancer-From Prevention to Patient Care, InTech, Rijeka, pp. 211-226.

http://www.intechopen.com/books/colorectal-cancer-fro m-prevention-to-patient-care/effects-of-dietary-counselin g-on-patients-with-colorectal-cancer

[2] D. Mayers, "Colon Polyps and Cancer Risk," 2007. http://coloncancer.about.com/od/screening/a/Polyps.htm

[3] P. W. Carne, J. N. Frye, A. Kennedy-Smith, J. Keating, A. Merrie, E. Dennett and F. A. Frizelle, "Local Invasion of the Bladder with Colorectal Cancers: Surgical Management and Patterns of Local Recurrence," Disease of Colon Rectum, Vol. 47, No. 1, 2004, pp. 44-47. doi:10.1007/s10350-003-0011-Z

[4] H. Ueno, C. Yamauchi, K. Hase, T. Ichikura and H. Mochizuki, "Clinicopathological Study of Intrapelvic Cancer Spread to the Iliac Area in Lower Rectal Adenocarcinoma by Serial Sectioning," British Journal of Surgery, Vol. 86, No. 12, 1999, pp. 1532-1537. doi:10.1046/j.1365-2168.1999.01271.x

[5] M. Astin, T. Griffin, R. D. Neal, P. Rose and W. Hamilton, "The Diagnostic Value of Symptoms for Colorectal Cancer in Primary Care: A Systematic Review," The British Journal of General Practice: The Journal of the Royal College of General Practitioners, Vol. 61, No. 586, 2011, pp. 231-243.

[6] P. Cervera and J. F. Fléjou, "Changing Pathology with Changing Drugs: Tumors of the Gastrointestinal Tract," Pathobiology, Vol. 78, No. 2, 2011, pp. 76-89. doi:10.1159/000315535

[7] M. S. Cappell, "Pathophysiology, Clinical Presentation, and Management of Colon Cancer," Gastroenterology Clinics of North America, Vol. 37, No. 1, 2008, pp. 1-24. doi:10.1016/j.gtc.2007.12.002

[8] A. P. Wimmer, J. P. Bouffard, P. R. Storms, J. A. Pilcher, 
C. Y. Liang and J. J. DeGuide, "Primary Colon Cancer without Gross Mucosal Tumor: Unusual Presentation of a Common Malignancy," Southern Medical Journal, Vol. 91, No. 12, 1998, pp. 1173-1176. doi:10.1097/00007611-199812000-00017

[9] K. J. d'Silva, A. J. Dwivedi, A. Shetty and S. Prakash, "An Unusual Presentation of Colon Cancer in a Young Individual," Digestive Diseases and Sciences, Vol. 50, No. 6, 2005, pp. 1033-1035. doi:10.1007/s10620-005-2699-1

[10] H. Wong, K. S. Law, P. Chan and T. Yau, "An Unusual Presentation of a Colon Cancer Patient Case Report," Cancer Therapy, Vol. 6, 2008, pp. 783-786.

[11] E. W. Toh, B. Griffiths and M. Farooq, "An Atypical Presentation of Colorectal Cancer," Journal of Surgical
Case Reports, Vol. 1, 2012, p. 2.

[12] F. Fahrtash, D. Chan, A. Colebatch and J. Rutovitz, "A Very Unusual Presentation of Metastatic Colon Cancer," ISRN Oncology, Vol. 2011, 2011, Article ID: 531803.

[13] L. K. Tumwine, M. Kagimu, P. Ocama, I. Segamwenge, N. Masiira-Mukasa, D. Wamala, O. Dworak and C. K. Opio, "Atypical Presentation of Colon Adenocarcinoma: A Case Report," Journal of Medical Case Reports, Vol. 6, 2012, p. 58. doi:10.1186/1752-1947-6-58

[14] F. Cellini and V. Valentini, "Current Perspectives on Preoperative Integrated Treatments for Locally Advanced Rectal Cancer: A Review of Agreement and Controversies," Oncology (Williston Park), Vol. 26, No. 8, 2012, pp. 730-735, 741. 